\title{
FATORES QUE PODEM CONTRIBUIR PARA TRANSTORNOS PSIQUIÁTRICOS NOS HABITANTES DE CARDOSO MOREIRA-RJ
}

\author{
Fernanda Caetano de Lucas Marques Talão ${ }^{I}$, Juliany Carvalho de Souza ${ }^{1}$, Maria Clara \\ Campos Alves ${ }^{I}$, Francisco de Assis Léo Machado ${ }^{12 *} \&$ Aldo Shimoya ${ }^{I}$
}

TALÃO, F.C.L.M.; SOUZA, J.C.; ALVES, M.C.C.; MACHADO, F.A.L.; SHIMOYA, A. Fatores que podem contribuir para transtornos psiquiátricos nos habitantes de Cardoso Moreira-RJ. Perspectivas Online: Biológicas \& Saúde, v.10, n.32, p.36-50, 2020.

\section{RESUMO}

Com o aumento da procura por serviços psiquiátricos em uma sociedade considerada estressada, não há como ignorar os fatores semelhantes aos indivíduos que sofrem de algum transtorno mental. Através da Estratégia de Saúde da Família, do Sistema Único de Saúde brasileiro, é possível identificar e avaliar os fatores, comuns a esses indíviduos, que podem contribuir para o acometimento desses transtornos na população. $\mathrm{O}$ objetivo deste trabalho é identificar e avaliar os fatores que podem contribuir para transtornos psiquiátricos nos habitantes de Cardoso Moreira-RJ. Os dados foram obtidos a partir da aplicação de 240 questionários pelos Agentes Comunitários de Saúde na microárea de Cardoso Moreira-RJ. Para a análise dos dados foi utilizada a Estatística Descritiva e os resultados apresentados por meio de tabelas e gráficos. Nesse estudo evidenciou-se que $10 \%$ da amostra fez/faz tratamento psiquiátrico. Além disso, os fatores, identificados nesses indivíduos, mais prevalentes que podem contribuir para o surgimento de transtornos psiquiátricos foram: tabagismo, diabetes, doenças cardíacas, problema nos rins, asma, internações médicas e animais de estimação em domicilio.

Palavras-chave: Transtorno Mental Comum; Sistema Único de Saúde; Estratégia de Saúde da Família. 


\begin{abstract}
Given the increasing demand for psychiatric services in a society considered to be stressed, there is no way to ignore similar factors that affect individuals who suffer from a mental disorder. By using data obtained from the Estratégia de Saúde da Família (Family Health Strategy), which is a strategy of the Sistema Único de Saúde (Brazilian Unified Health System) in Brazil, it is possible to identify and evaluate possible factors that may contribute to the occurrence of these disorders in the population. The objective of the work is to identify and evaluate the

disorders in the inhabitants of Cardoso Moreira-RJ. Data were collected from 240 questionnaires applied by the Community Health Worker. Descriptive statistics were used in the methodology. Results were displayed using tables and graphs. In this study, a considerably high percentage was evidenced in people such as: smokers, diabetes, heart disease, kidney problems, asma, medical admissions and pet at home. All the factors researched in this work are related to Common Mental Disorder, however the pregnancy was represented by only one person.
\end{abstract} factors that may contribute to psychiatric

Keywords: Common Mental Disorder; Brazilian Unified Health System; Family Health Strategy.

\footnotetext{
${ }^{1}$ Universidade Cândido Mendes - UCAM-CAMPOS - Rua Anita Pessanha, 100, Parque São Caetano, Campos dos Goytacazes, RJ, CEP: 28030-335, Brasil.

${ }^{2}$ Institutos Superiores de Ensino do CENSA - ISECENSA - Rua Salvador Correa, 139, Centro, Campos dos Goytacazes, RJ, CEP: 28035-310, Brasil.

(*) e-mail: franciscoleomachado@gmail.com

Data de recebimento: 05/11/2019. Aceito para publicação: 21/02/2020.
}

Persp. online: biol. \& saúde, Campos dos Goytacazes, 32 (10),36-50, 2020

seer.perspectivasonline.com.br 


\section{INTRODUÇÃO}

Diversos grupos de pesquisa em todo mundo apontam transtornos mentais associados a vários tipos de doenças que a população vem enfrentando. Segundo Skapinakis et al. (2013), nos últimos 30 anos, estudos epidemiológicos têm demonstrado, que os transtornos mentais representam aproximadamente $12 \%$ da carga global de doenças ocasionando uma das principais fontes de incapacidade.

Para Vos et al. (2012) dentre todos os distúrbios, os transtornos de ansiedade e/ou depressão, também conhecidos como Transtornos Mentais Comuns (TMC), são os mais importantes do ponto de vista da saúde pública devido à sua prevalência e persistência. Esse pensamento foi corroborado por Silva et al. (2018) ao afirmar que o TMC é considerado o sofrimento mais prevalente na população mundial.

Além dos transtornos acima citados, Vidal et al. (2014) afirmaram que sintomas como: dificuldades de concentração, insônia, irritabilidade, esquecimento, fadiga e queixas de somatização são frequentes na população global. Os transtornos por uso de álcool também estão na lista das vinte principais causas de incapacidade em todo o mundo (EATON et. al., 2008; Vos et al., 2012) uma vez que o álcool é apontado como a substância psicoativa mundialmente mais consumida (Nelson e McNall, 2016). Munhoz et al. (2017) relataram que a Organização Mundial da Saúde (OMS, 2010) estimou que no ano de 2009, 38,3\% da população mundial com idade $\geq 15$ anos haviam consumido bebidas alcoólicas.

Melo et al. (2017) alertaram que o estudo referente a Carga Global de Doenças (Global Burden of Diseases- GBD) de 2015 indicava que de 1990 a 2015, o consumo excessivo de álcool foi um dos fatores de risco que mais contribuíram no mundo para anos de vida perdidos por morte ou incapacidade (Disability-Adjusted LifeYears - DALYs) e que no Brasil, no ano de 2015, o álcool ocupava a quinta posição no ranking dos fatores de risco que mais contribuíram para o total de DALYs, tanto para o sexo masculino quanto feminino.

Em função destas discussões se torna importante que os países criem sistemas nacionais para melhor gerir diversos problemas de saúde, dentre eles, transtornos mentais comuns ou uso de substâncias nocivas. No Brasil, existe o Sistema Único de Saúde (SUS) que criou a Estratégia de Saúde da Família (ESF) como forma de política nacional de atenção básica (PINTO; GIOVANELLA, 2018).

O ESF é formado por uma equipe multidisciplinar em que o agente comunitário de saúde (ACS) é o integrante de maior contato com a população. Esse profissional dispõe de fichas de cadastro familiar e individual que são preenchidas durante visitas domiciliares em uma determinada microárea. Os dados contidos nessas fichas têm caráter biológico e socioeconômico. Bandeira, Freitas e Carvalho Filho (2007) relataram efeito positivo do Programa de Saúde da Família (PSF), atualmente remodelado como Estratégia de Saúde da Família (ESF), em indicadores de saúde da população obtidos de estudos isolados realizados em municipios brasileiros.

A partir de alguns desses dados há a identificação de possíveis pessoas portadoras de algum sintoma de transtorno psiquiátrico. O presente trabalho buscou identificar e avaliar os fatores que podem contribuir para transtornos psiquiátricos nos habitantes de Cardoso Moreira-RJ. 


\section{METODOLOGIA}

A pesquisa foi realizada em uma microárea pré-estabelecida na cidade de Cardoso Moreira-RJ, que possui cerca de 309 pacientes cadastrados. Foi desenvolvido um estudo descritivo, quantitativo e transversal utilizando dados extraídos das fichas de cadastramento da Estratégia de Saúde da Família como gênero, peso $(\mathrm{kg})$, itens relacionados à saúde como: gestante, fumante, diabetes, AVC/derrame, infarto, insuficiência cardíaca, doença cardíaca, insuficiência renal, problemas nos rins, asma, DPOC/Enfisema, problema respiratório, câncer, e internação nos últimos 12 meses. Além disso, foram verificadas as condições de vida dos usuários tais como: situação de moradia, número de moradores no domicílio, número de cômodos do domicílio.

A principal função de um Agente Comunitário de Saúde é realizar o cadastramento de todos os indivíduos das famílias de uma microárea pré-estabelecida, devendo manter esse cadastro atualizado para o acompanhamento de saúde de cada indivíduo. Para tal, é utilizada uma ficha de cadastro individual e familiar que contém perguntas de caráter biológico e socioeconômico.

As fichas fazem parte do Sistema com Coleta de Dados Simplificada (CDS), um software transitório usado para captação de dados por meio de sete fichas de registro. Tal sistema faz parte do e-SUS Atenção Primária (e-SUS APS), ou seja, o processo de informatização qualificada do SUS em busca de um SUS eletrônico. Além do e-SUS CDS há o e-SUS PEC - Prontuário Eletrônico do Cidadão - disponível em UBS que foram informatizadas pelo Departamento de Atenção Básica (BRASIL, 2020).

Para calcular o tamanho da amostra foi utilizada a equação proposta por GIL (2008).

$$
\mathrm{n}=\frac{\sigma^{2} \cdot \mathrm{p} \cdot \mathrm{q} \cdot \mathrm{N}}{\mathrm{e}^{2}(\mathrm{~N}-1)+\sigma^{2} \cdot \mathrm{p} \cdot \mathrm{q}}
$$

onde:

$\mathrm{n}=$ tamanho da amostra $(\cong 240)$

$\sigma=$ nível de confiança escolhido, expresso em número de desvio-padrão $(=1,96)$

$\mathrm{p}=$ percentagem com a qual o fenômeno se verifica $(=0,5)$

$\mathrm{q}=$ percentagem complementar $(100-\mathrm{p})(=0,5)$

$\mathrm{N}=$ tamanho da população $(=309)$

$\mathrm{e}=$ erro máximo permitido $(=0,03)$

Adotou-se um nível de significância de $5 \%$ de probabilidade, com margem de erro de $3 \%$, dessa forma foram entrevistados 240 cidadãos com idade mínima de 18 anos.

Os dados obtidos por meio da aplicação do questionário foram tabulados utilizando a planilha do Excel. Utilizou-se a estatística descritiva para obtenção de frequências, médias e erro padrão dos itens do questionário. Os resultados foram apresentados por meio de tabelas e gráficos.

\section{RESULTADOS E DISCUSSÃO}

O item de maior importância e que serviu de comparação para identificar o TMC com os demais itens foi se o entrevistado fez ou faz tratamento com médico psiquiatra. Em relação 
a essa pergunta, $10 \%$ do total de 240 entrevistados responderam SIM.

Na Figura 1 é possível observar a distribuição dos indivíduos em três grupos distintos que fizeram/fazem tratamento psiquiátrico: gestantes ( 1 indivíduo), fumantes (30 indivíduos) e diabéticos (16 indivíduos). No grupo de gestantes nunca fez/faz tratamento com psiquiatras, dentre os fumantes (17\%) enquanto no grupo dos diabéticos $19 \%$ das pessoas entrevistadas fizeram/fazem tratamento com psiquiatra.

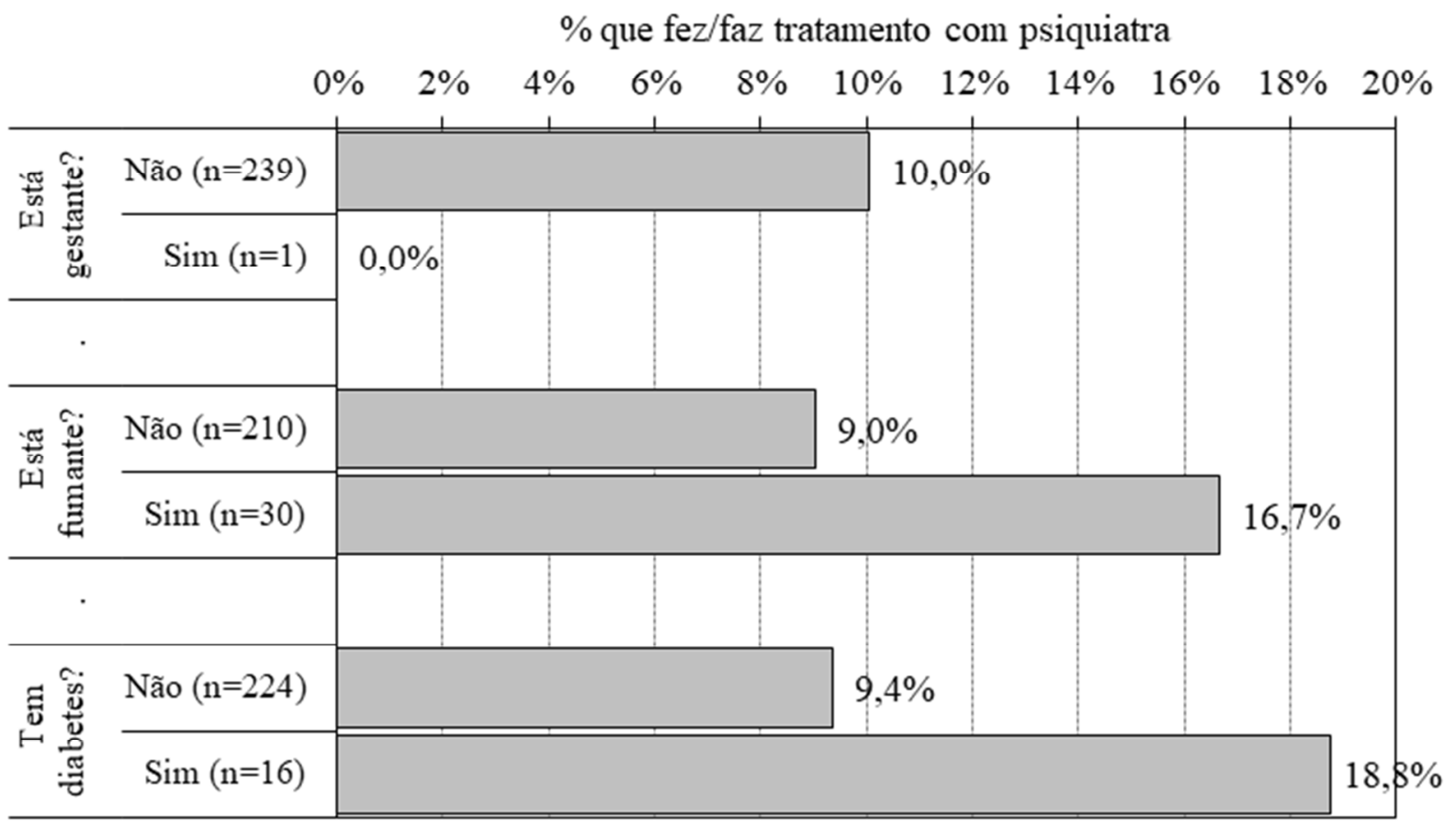

Figura 1 - Porcentagens de pessoas que fizeram/fazem tratamento psiquiátrico por grupos de gestante, fumante e diabético.

Embora tenha sido entrevistado apenas uma gestante, Lucchese et al. (2017) relataram a prevalência de probabilidade entre gestação e TMC associada com as variáveis estado civil, idade gestacional, gravidez planejada e sangramento.

Observa-se que há uma porcentagem consideravelmente alta para os tabagistas, que pode estar associada a transtorno depressivo, corroborando com relatos de Rondina, Gorayeb e Botelho (2003). Entrevistados relatam que o uso do tabaco é uma espécie de auxílio para amenizar uma situação de estresse e combater a ansiedade, já que se trata de uma droga estimulante do sistema nervoso central.

A porcentagem de diabéticos que procuram tratamento psiquiátrico também é considerável. Henna, Salesse e Santos (2018) relatam uma associação entre diabetes e transtornos psiquiátricos, dificultando a adesão ao tratamento, controle glicêmico, levando ao aparecimento de complicações.

Na Figura 2 os indivíduos estão distribuídos em diferentes grupos: AVC/derrame (10), infarto (6), insuficiência cardíaca (1) e outra doença cardíaca (20). Verifica-se dentre o grupo outra doença cardíaca que $20 \%$ delas fizeram/fazem tratamento com psiquiatra seguido pelo grupo AVC/derrame com 10\%. Já nos grupos de infarto e insuficiência cardíaca ninguém fez/faz tratamento com psiquiatra. 
$\%$ que fez/faz tratamento com psiquiatra

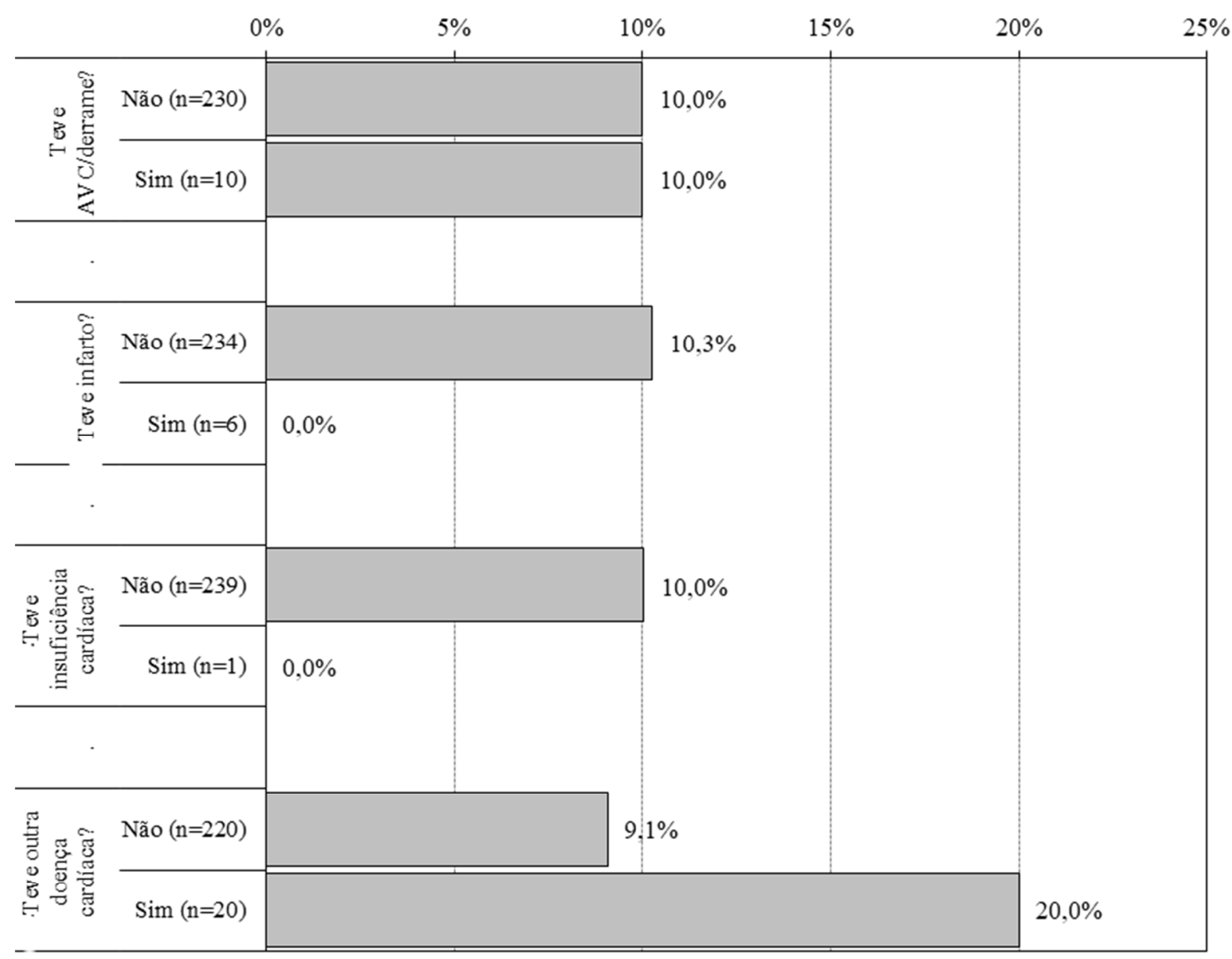

Figura 2 - Porcentagens de pessoas que fizeram/fazem tratamento psiquiátrico por grupos de AVC/derrame, infarto, insuficiência cardíaca e outra doença cardíaca.

Com relação às pessoas que apresentaram problemas cardíacos Nasser et al. (2016) relatam que a depressão e ansiedade são altamente prevalentes em indivíduos com doença arterial coronária e doenças cardiovasculares.

Na Figura 3 a distribuição dos indivíduos dá-se por grupos: insuficiência renal (2) ou outro problema renal (29). Pode-se observar que apesar dos indivíduos com insuficiência renal nunca terem feito tratamento psiquiátrico os demais que não tiveram insuficiência fizeram/fazem tratamento psiquiátrico. Já pessoas que tiveram outro problema nos rins, $20,7 \%$ delas fizeram/fazem tratamento com psiquiatra.

A insuficiencia renal crônica segundo Azevedo, Azevedo e Manhães (2015) é uma doença assintomática que pode acompanhar o indivíduo por muitos anos e só se manifestar quando ele já perdeu quase totalmente o funcionamento dos rins, em torno de $80 \%$, sem condições de reverter o quadro. De acordo com Moreira et al. (2014) os sintomas neuropsiquiátricos são frequentemente associada à disfunção renal e pode comprometer negativamente o curso clínico bem como a qualidade de vida, e a funcional situação dos pacientes. 
$\%$ que fez/faz tratamento com psiquiatra

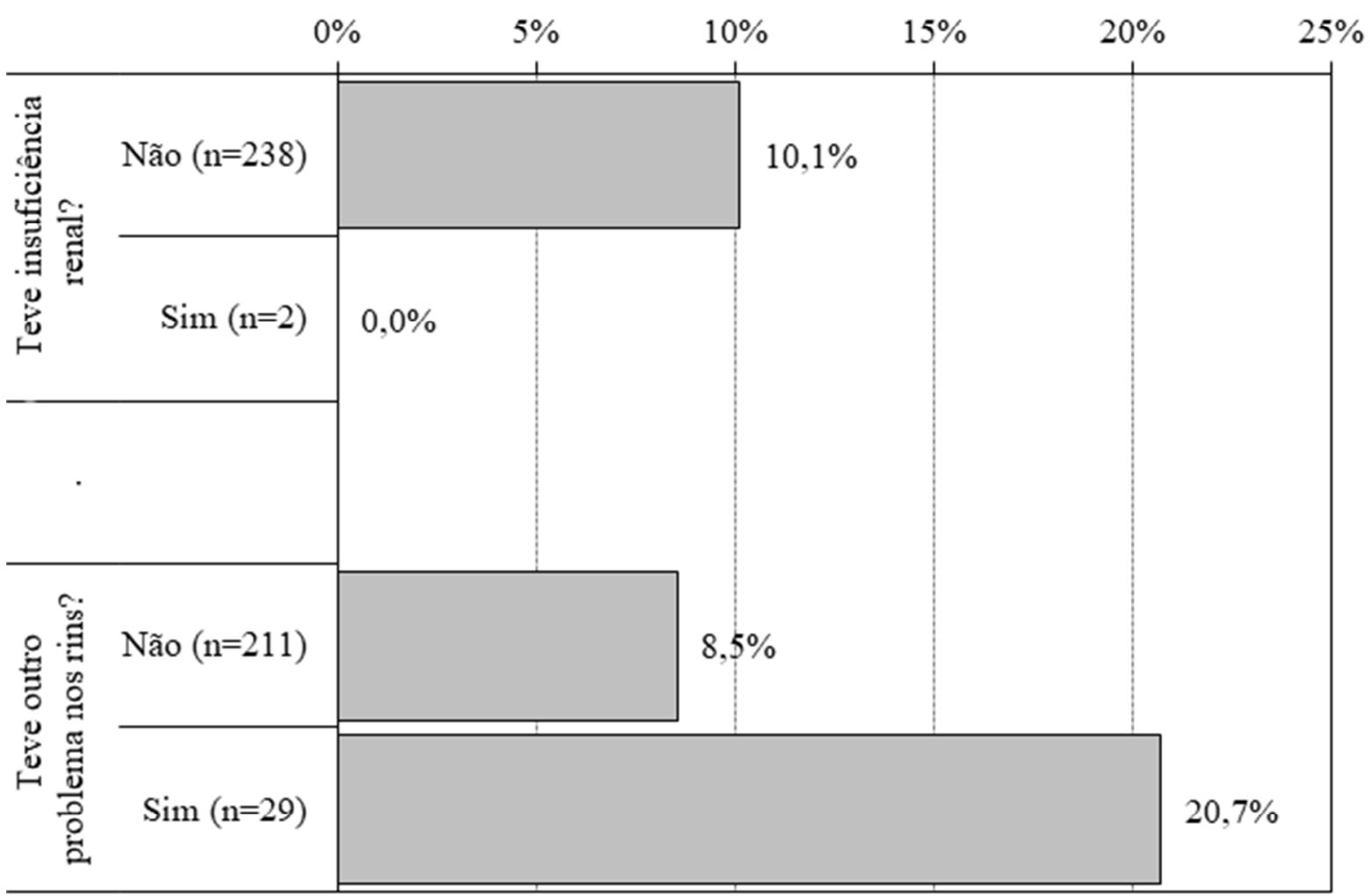

Figura 3 - Porcentagens de pessoas que fizeram/fazem tratamento psiquiátrico por grupos de insuficiência renal ou outro problema renal.

Na Figura 4 são apresentadas as distribuições dos indivíduos pelos grupos asma (2), DPOC//Enfisema (1) e outro problema respiratório (5). Nota-se que no grupo asma 50\% fez/faz tratamento psiquiátrico. Uma pessoa apenas tem doença pulmonar obstrutiva crônica (DPOC) /enfisema e ela não fez ou faz tratamento com psiquiatra, das pessoas que não tem DPOC/enfisema $10 \%$ fizeram/fazem tratamento com psiquiatra. Cinco pessoas têm outro problema respiratório, mas nenhuma delas fizeram/fazem tratamento com psiquiatra, das que não têm outro problema respiratório 10,2\% fizeram/fazem tratamento com psiquiatra. 
$\%$ que fez/faz tratamento com psiquiatra

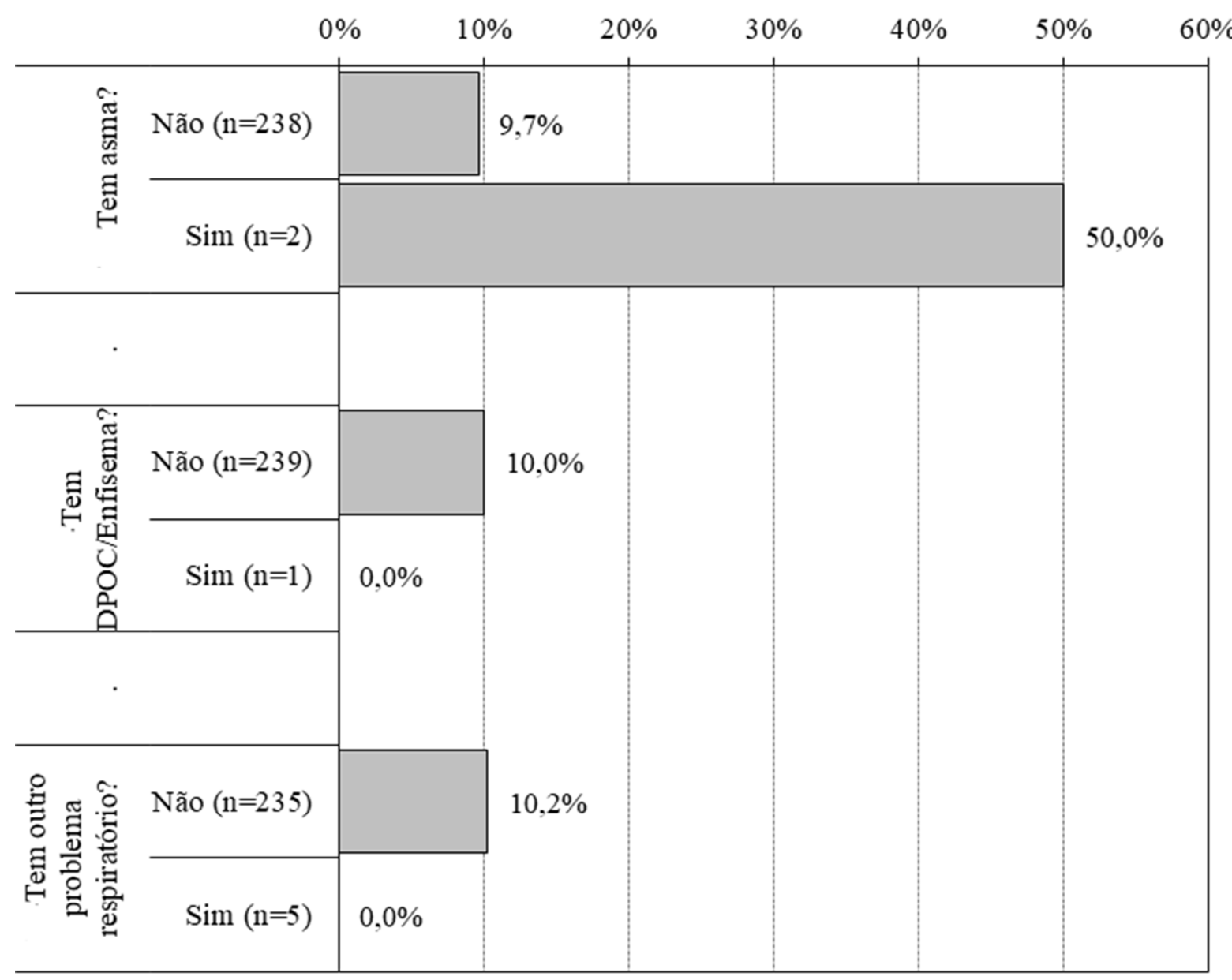

Figura 4 - Porcentagens de pessoas que fizeram/fazem tratamento psiquiátrico por grupos de asma, DPOC/Enfisema e outro problema respiratório.

Segundo Souza et al. (2016) a doença pulmonar obstrutiva crônica (DPOC) tem como característica a limitação do fluxo aéreo não totalmente reversível, progressiva e associada a uma resposta inflamatória anormal dos pulmões, à inalação de partículas ou gases nocivos. É uma das principais causas de morbidade e mortalidade no mundo, impondo substancial sobrecarga econômica sobre o indivíduo portador da doença e o sistema de saúde.

Pode-se observar na Figura 5 as porcentagens de pessoas que fizeram/fazem tratamento psiquiátrico por grupos de câncer (11) e alguma internação nos últimos 12 meses (30). Verifica-se no grupo câncer que as pessoas que têm ou tiveram câncer não fizeram/ fazem tratamento com psiquiatra, das que nunca tiveram câncer 10,5\% fizeram/fazem tratamento com psiquiatra. No grupo de internações nos últimos 12 meses, $20 \%$ delas fizeram/fazem tratamento com psiquiatra enquanto aquelas que não estiveram internadas, $8,6 \%$ fizeram/fazem tratamento com psiquiatra.

Segundo Santos (2017) o diagnóstico do câncer tem sido consistentemente associado a diversos transtornos mentais que podem potencializar as tendências autodestrutivas. $\mathrm{O}$ mesmo ainda relata a despeito da alta prevalência de transtornos depressivos em pacientes com câncer, essa temática permanece ainda pouco explorada. 


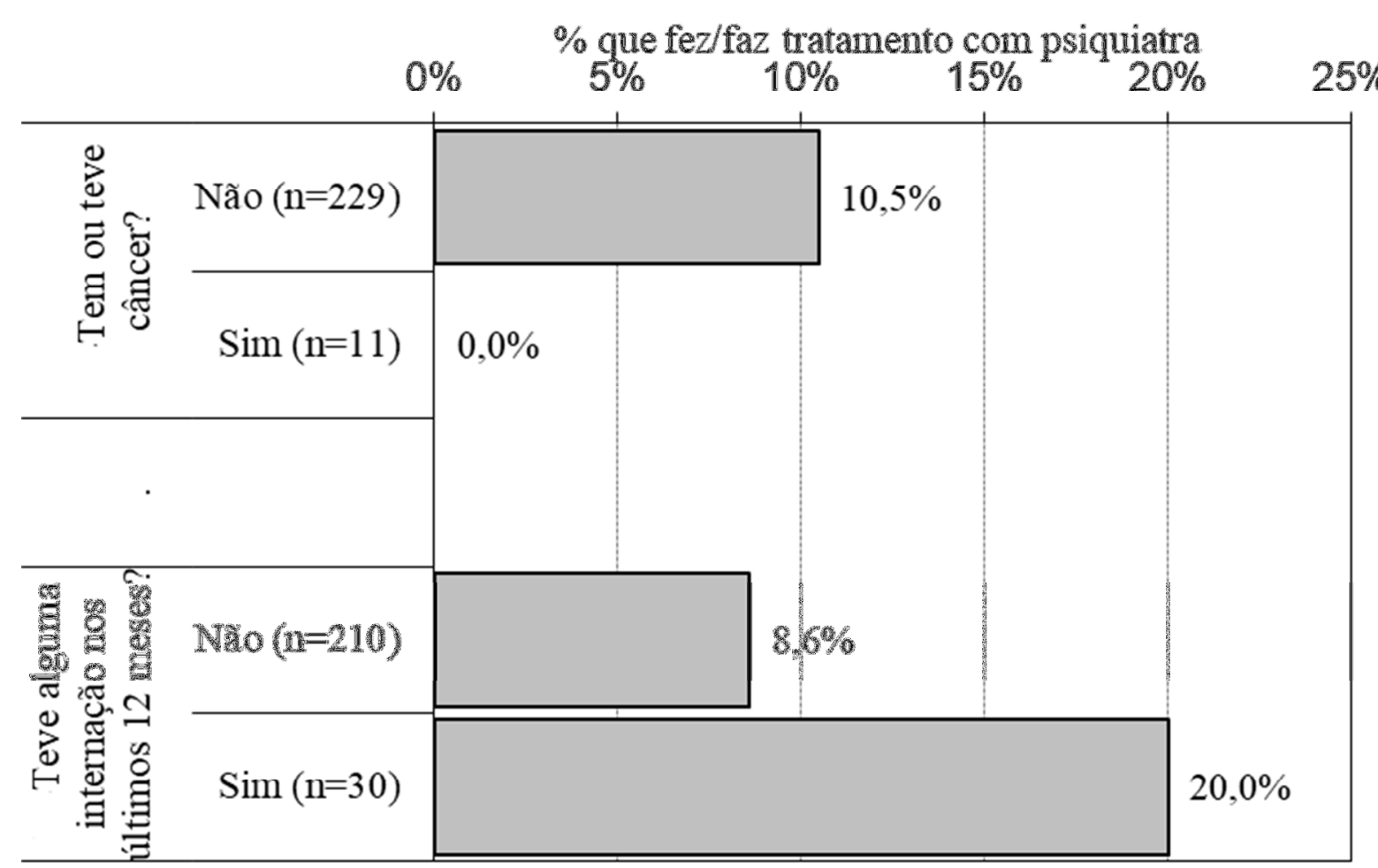

Figura 5 - Porcentagens de pessoas que fizeram/fazem tratamento psiquiátrico por grupos de câncer e alguma internação nos últimos 12 meses.

Na Figura 6 observa-se a distribuição dos indivíduos nos grupos de pessoas que possuem gato (18), cachorro (85), pássaro (30) ou animal de criação no domicílio (8). Verifica-se que nenhuma das pessoas com gato de estimação no domicílio fez/faz tratamento com psiquiatra, enquanto $10,8 \%$ das que não têm gato fizeram/fazem tratamento com psiquiatra. Das pessoas com cachorro de estimação no domicílio, 5,9\% fizeram/fazem tratamento com psiquiatra e das que não possuem 12,3\% fizeram/fazem tratamento com psiquiatra. No grupo das pessoas que têm pássaro de estimação no domicílio, nenhuma delas fez/faz tratamento com psiquiatra enquanto as que não possuem pássaro, 11,4\% fizeram/fazem tratamento com psiquiatra. Do grupo de pessoas que possuem animal de criação (porco, galinha) no domicílio 12,5\% fizeram/fazem tratamento com psiquiatra e das que não possuem $10 \%$ fizeram/fazem tratamento com psiquiatra.

Estudos relatam que animais de estimação ajudam a lidar com depressão, ansiedade e estresse, ou seja, os principais Transtornos Mentais Comuns (TMC). Além disso, pesquisas feitas pela Associação Americana de Saúde do Coração afirmam que o convívio com pets diminui o risco de doenças cardíacas (BEZERRA, 2018). 


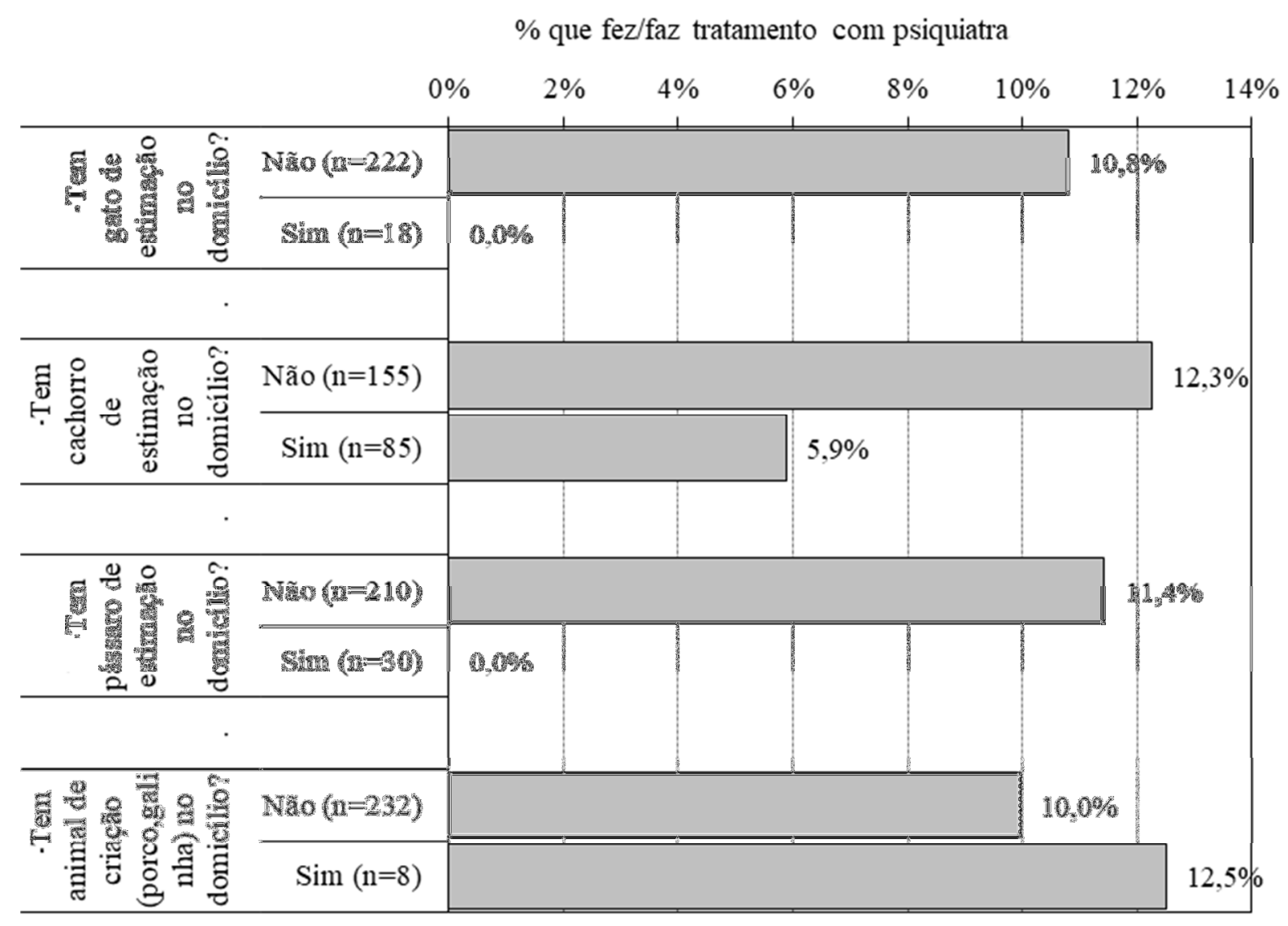

Figura 6 - Porcentagens de pessoas que fizeram/fazem tratamento psiquiátrico por grupos de ter gato, cachorro, pássaro ou animal de criação no domicílio.

Nota-se na Figura 7 a distribuição dos indivíduos por grupos de situação de moradia, gênero, número de moradores no domicilio, número de cômodos do domicilio e como se consideram em relação ao seu peso.

Observa-se que 155 pessoas têm moradia própria e 11,6\% delas fizeram/fazem tratamento com psiquiatra; 62 pessoas têm moradia alugada e 4,8\% delas fizeram/fazem tratamento com psiquiatra. Das 13 pessoas com moradia cedida, $15,4 \%$ delas fizeram ou fazem tratamento com psiquiatra. E das 10 pessoas com outra situação de moradia, $10 \%$ delas fizeram/fazem tratamento com psiquiatra. Do total de pessoas entrevistadas, 123 são do gênero feminino e 10,6\% fizeram/fazem tratamento com psiquiatra. As demais 127 pessoas do gênero masculino apresentaram $9,4 \%$ que fizeram/fazem tratamento com psiquiatra.

De acordo com a literatura citada nesta pesquisa é notado que o questionário usado não é compatível com os demais. Gonçalves; Kapczinski (2008) e Maragno et al. (2006), utilizaram o Self-Reporting Questionnaire, recomendado pela Organização Mundial da Saúde, (OMS), em que o único dado que corresponde é o item gênero. Na obra dos autores citados, mulheres apresentaram maior chance de apresentar TMC, em concordância com o presente trabalho. Bandeira; Freitas; Carvalho Filho (2007) usou o a Escala de Saúde Geral de Goldberg (QSG) e validada para o Brasil por Pasquali et al. (1994). Os autores encontraram em seu trabalho que os homens têm um perfil sintomático maior que as mulheres. 
Em relação Trinta e sete pessoas moram sozinhas e $21,6 \%$ delas fizeram/fazem tratamento com psiquiatra. Pessoas que moram com mais um morador são 45 e 8,9\% fizeram/fazem tratamento com psiquiatra. Oitenta pessoas têm moradia em três pessoas e 5\% delas fizeram ou fazem tratamento com psiquiatra. Cinquenta e uma pessoas moram em moradias com quatro moradores e $15,7 \%$ delas fizeram ou fazem tratamento com psiquiatra. Vinte e sete pessoas moram em cinco ou mais pessoas e nenhuma delas fez ou faz tratamento com psiquiatra. Pessoas com moradia de até quatro cômodos são quinze e 13,3\% delas fizeram ou fazem tratamento com psiquiatra. Com moradia de cinco cômodos são cinquenta e cinco pessoas e $9,1 \%$ delas fizeram ou fazem tratamento com psiquiatra. Com seis cômodos na moradia são trinta e oito pessoas e $7,9 \%$ delas fizeram ou fazem tratamento com psiquiatra. Sessenta e duas pessoas moram em moradias de sete cômodos e 9,7\% delas fizeram ou fazem tratamento com psiquiatra. Pessoas com moradia de oito cômodos são trinta e sete e 10,8\% delas fizeram ou fazem tratamento com psiquiatra. Trinta e três pessoas têm moradia com nove cômodos ou mais e 12,1\% fizeram ou fazem tratamento com psiquiatra. Para Furtado et al. (2013) a demanda por moradias de pessoas com transtorno mental é uma implicação fundamental da mudança do modelo de assistência em saúde mental no Brasil, uma vez que prioriza o direcionamento do cuidado para a comunidade, superando a centralidade do hospital psiquiátrico. As necessidades de moradia não devem ser subestimadas, uma vez que a mesma é vista como determinante social da saúde em geral e da saúde mental em particular, a moradia é decisiva na inserção, integração e manutenção de pessoas no meio social.

Pessoas que se consideram abaixo do peso são quatro e nenhuma delas fez ou faz tratamento com psiquiatra. Cento e quarenta e duas pessoas se consideram no peso adequado e 10,6\% delas fizeram ou fazem tratamento com psiquiatra. Por fim, noventa e quatro pessoas se consideram acima do peso e 9,6\% delas fizeram ou fazem tratamento com psiquiatra. Segundo Nicolau, Espirito Santo e Polakiewicz (2017) a obesidade é doença e fator de risco, ela tem incidência direta e negativa na qualidade de vida dos indivíduos por ela acometidos, além disso, o seu tratamento deve ser centrado no sujeito e conduzido por uma equipe de saúde especializada, multiprofissional e com atuação interdisciplinar. Silva (2015) relatou que os resultados obtidos em seu estudo não demonstraram associação significativa entre sobrepeso e obesidade e transtornos mentais comuns. Esses achados são consistentes com alguns estudos realizados por outros autores em populações e contrários a outros autores que encontraram associação entre transtornos mentais e excesso de peso, especialmente obesidade e depressão. 


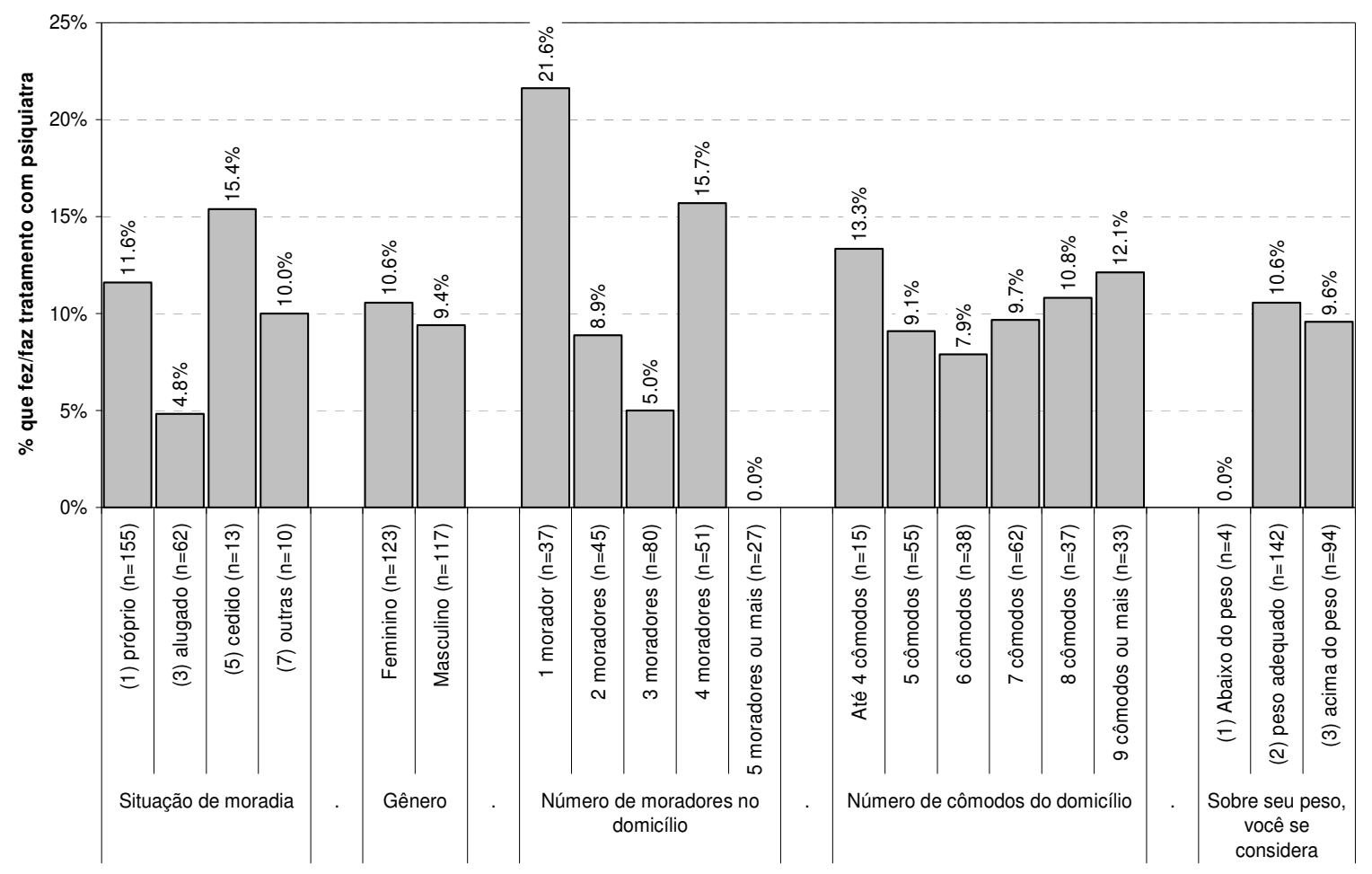

Figura 7 - Porcentagens de pessoas que fizeram/fazem tratamento psiquiátrico por grupos de situação de moradia, gênero, número de moradores no domicilio, número de cômodos do domicilio e como se considera em relação ao seu peso.

\section{CONCLUSÕES}

Nesse estudo evidenciou-se uma porcentagem consideravelmente elevada de pessoas que apresentam fatores que podem contribuir para o surgimento de Transtornos Mentais Comum (TMC), estas pessoas podem ser fumantes, apresentarem diabetes, doenças cardíacas, problema nos rins ou asma.

Pessoas que sofreram internações médicas e possuem animais de estimação em domicilio também estão associadas ao TMC. Vale ressaltar que animais em domicílios podem ser um atenuante ao TMC. Dentre todos os fatores pesquisados nesse trabalho que estão relacionados ao TMC, a gestação foi representada apenas por uma entrevistada.

\section{REFERÊNCIAS}

AZEVEDO, S. M.; AZEVEDO, A. S.; MANHÃES, L.S.P. Insuficiência renal crônica: análise do binômio enfermeiro-portador de IRC. Perspectivas online Biológicas \& Saúde, Campos dos Goytacazes, v. 5, n. 19, p.11-34, 2015. DOI: 10.25242/88685192015883

BANDEIRA, M.; FREITAS, L. C.; CARVALHO FILHO, J. G. T. Avaliação da ocorrência de transtornos mentais comuns em usuários do Programa de Saúde da Família. Jornal Brasileiro de Psiquiatria, [s.1.], v. 56, n. 1, p. 41-47, 2007. DOI: 10.1590/S004720852007000100010 
BEZERRA, K. Animais de estimação ajudam a lidar com depressão, ansiedade e estresse. Disponível em: <https://www.clubeparacachorros.com.br/tabombando/animais-deestimacao-ajudam-lidar-com-depressao-ansiedade-e-estresse/>. Acesso em: 24 nov. 2018.

EATON, W. W.; MARTINS, S.S.; NESTADT, G.; BIENVENU, O.J.; CLARKE, D.; ALEXANDRE, P. The Burden of Mental Disorders. Epidemiologic Reviews, [s.1.], v. 30, n. 1, p.1-14, 14 maio 2008. DOI: 10.1093/epirev/mxn011

FURTADO, J. P.; TUGNY, A.; BALTAZAR, A.P.; KAPP, S.; GENEROSO, C.M.; CAMPOS, F.C.B.; GUERRA, A.M.C.; NAKAMURA, E. Modos de morar de pessoas com transtorno mental grave no Brasil: uma avaliação interdisciplinar. Ciência \& Saúde Coletiva, Rio de Janeiro, v. 18, n. 12, p.3683-3693, 2013.

GIL, A. C. Métodos e Técnicas de Pesquisa Social. 6. ed. São Paulo: Atlas. 2008. 200p.

GONÇALVES, D. M.; KAPCZINSKI, F. Transtornos mentais em comunidade atendida pelo Programa Saúde da Família. Cadernos de Saúde Pública, Rio de Janeiro, v. 24, n. 7, p. 1641-1650, jul. 2008. DOI: 10.1590/S0102-311X2008000700019

HENNA, E.A.D.; SALESSE, M.T.; SANTOS, M.M. Prevalência de transtorno mental comum e transtorno de personalidade em pacientes com diabetes mellitus tipo 1 atendidos no ambulatório do Conjunto Hospitalar de Sorocaba-SP. In: CONGRESSO DA SUMEP, 35. 2018, Sorocaba. Anais. Sorocaba: Revista da Faculdade de Ciências Médicas de Sorocaba, 2018. v. 20, p. 1 - 1. Disponível em: <https://revistas.pucsp.br/RFCMS/article/view/40091>. Acesso em: 20 jul. 2019.

LUCCHESE, R.; SIMÕES, N.D.; MONTEIRO, L.H.B.; VERA, I.; FERNANDES, I.L.; CASTRO, P.A.; SILVA, G.C.; EVANGELISTA, R.A.; BUENO, A.A.; LEMOS, M.F. Factors associated with the probability of common mental disorders in pregnant women: a cross-sectional study. Escola Anna Nery, Rio de Janeiro, v. 21, n. 3, p.1-6, 2017. DOI: 10.1590/2177-9465-ean-2016-0094

MARAGNO, L.; GOLDBAUM, M.; GIANINI, R.J.; NOVAES, H.M.D.; CÉSAR, C.L.G. Prevalência de transtornos mentais comuns em populações atendidas pelo Programa Saúde da Família (QUALIS) no Município de São Paulo, Brasil. Cadernos de Saúde Pública, Rio de Janeiro, v. 22, n. 8, p. 1639-1648, ago. 2006.

MELO, A.P.S.; FRANÇA, E.B.; MALTA, D.C.; GARCIA, L.P.; MOONEY, M.; NAGHAVI, M. Mortalidade por cirrose, câncer hepático e transtornos devidos ao uso de álcool: Carga Global de Doenças no Brasil, 1990 e 2015. Revista Brasileira de Epidemiologia, São Paulo, v. 20, n. 1, p.61-74, 2017. DOI: 10.1590/1980-5497201700050006

MOREIRA, J.M.; MATTA, S.M.; KUMMER, A.M.; BARBOSA, I.G.; TEIXEIRA, A.L.; SILVA, A.C.S. Neuropsychiatric disorders and renal diseases: an update. Jornal Brasileiro de Nefrologia, São Paulo, v. 36, n. 3, p. 396-400, 2014. DOI: 10.5935/0101-2800.20140056

MUNHOZ, T.N.; SANTOS, I.S.; NUNES, B.P.; MOLA, C.L.; SILVA, I.C.M.; MATIJASEVICH, A. Tendências de consumo abusivo de álcool nas capitais brasileiras entre os anos de 2006 a 2013: análise das informações do VIGITEL. Cadernos de Saúde Pública, Rio de Janeiro, v. 33, n. 7, p.1-11, 2017. DOI: 10.1590/0102-311X00104516 
NASSER, F.J.; ALMEIDA, M.M.; SILVA, L.S.; ALMEIDA, R.G.P.; BARBIRATO, G.B.; MENDLOWICZ, M.V.; MESQUITA, C.T. Psychiatric Disorders and Cardiovascular System: Heart-Brain Interaction. International Journal Of Cardiovascular Sciences, [s.1.], v. 29, n. 1, p.65-75, 2016. DOI: 10.5935/2359-4802.20160003

NELSON, J.P.; MCNALL, A.D. Alcohol prices, taxes, and alcohol-related harms: A critical review of natural experiments in alcohol policy for nine countries. Health Policy, v. 120, n. 3, p.264-272, 2016. DOI: 10.1016/j.healthpol.2016.01.018

NICOLAU, I.R.; ESPÍRITO SANTO, F.H.; POLAKIEWICZ, R.R. Hipertensão em pacientes acompanhados em um centro de referência em obesidade. Perspectivas online Biológicas \& Saúde, Campos dos Goytaczes, v. 7, n. 23, 2017. DOI: 10.25242/88687232017722

PASQUALI, L.; GOUVEIA, V.V.; ANDRIOLA, W.B.; MIRANDA, F.J.; RAMOS, A.L.M. Questionário de Saúde Geral de Goldberg (QSG): Adaptação brasileira. Psicologia: Teoria e Pesquisa, Brasília, v. 10, n. 3, p. 421-437, 1994.

PINTO, L.F.; GIOVANELLA, L. Do Programa à Estratégia Saúde da Família: expansão do acesso e redução das internações por condições sensíveis à atenção básica (ICSAB). Ciência \& Saúde Coletiva, Rio de Janeiro, v. 23, n. 6, p.1903-1914, 2018. DOI: 10.1590/141381232018236.05592018

RONDINA, R.C.; GORAYEB, R.; BOTELHO, C. Relação entre tabagismo e transtornos psiquiátricos. Archives Of Clinical Psychiatry (São Paulo), São Paulo, v. 30, n. 6, p.221228, 2003. DOI: 10.1590/S0101-60832003000600005

SANTOS, M.A. Câncer e suicídio em idosos: determinantes psicossociais do risco, psicopatologia e oportunidades para prevenção. Ciência \& Saúde Coletiva, [s.1.], v. 22, n. 9, p.3061-3075, set. 2017. DOI: 10.1590/1413-81232017229.05882016

SILVA P.A.S.; ROCHA, S.V.; SANTOS, L.B.; SANTOS. C.A.; AMORIM. C.R.; VILELA, A.B.A. Prevalência de transtornos mentais comuns e fatores associados entre idosos de um município do Brasil. Ciências \& Saúde Coletiva. 2018; 23(2):639-46. DOI:10.1590/141381232018232.12852016

SILVA, T.M.; AGUIAR, O.B.; FONSECA, M.J.M. Associação entre sobrepeso, obesidade e transtornos mentais comuns em nutricionistas. Jornal Brasileiro de Psiquiatria, Rio de Janeiro, v. 64, n. 1, p.24-31, 2015. DOI: 10.1590/0047-2085000000053

SOUSA, C.A.; CÉSAR, C.L.G.; BARROS, M.B.A.; CARANDINA, L.; GOLDBAUM, M.; PEREIRA, J.C.R. Doenças respiratórias e fatores associados: estudo de base populacional em São Paulo, 2008-2009. Revista de Saúde Pública, São Paulo, v. 46, n. 1, p.16-25, 2012.

SKAPINAKIS, P.; BELLOS, S.; KOUPIDIS, S.; GRAMMATIKOPOULOS, I.; THEODORAKIS, P.N.; MAVREAS, V. Prevalence and sociodemographic associations of common mental disorders in a nationally representative sample of the general population of Greece. Bmc Psychiatry, [s.1.], v. 13, n. 1, p.1-14, 2013. DOI: 10.1186/1471-244X-13-163

VIDAL, C.E.L.; AMARA, B.; FERREIRA, D.P.; DIAS, I.M.F.; VILELA, L.A.; FRANCO, L.R. Preditores de prováveis transtornos mentais comuns (TMC) em prostitutas utilizando o 
Self-Reporting Questionnaire. Jornal Brasileiro de Psiquiatria, Rio de Janeiro, v. 63, n. 3, p. 205-212, 2014. DOI: 10.1590/0047-2085000000027

VOS, T.; FLAXMAN, A.D.; NAGHAVI, M.; LOZANO, R.; MICHAUD, C.; EZZATI, M.; SHIBUYA, K.; SALOMON, J.A.; ABDALLA, S.; ABOYANS, V.; ABRAHAM, J.; ACKERMAN, I.; AGGARWAL, R., AHN, S.Y.; ALI, M.K.; ALMAZRO, M.A.; ALVARADO, M.; ANDERSON, H.R.; MURRAY, C.J.L. Years lived with disability (YLDs) for 1160 sequelae of 289 diseases and injuries 1990-2010: a systematic analysis for the Global Burden of Disease Study 2010. The Lancet, [s.1.], v. 380, n. 9859, p.2163-2196, 2012. DOI: 10.1016/S0140-6736(12)61729-2 